\title{
Comprehensive Analysis of Differentially Expressed Profiles of IncRNAs/mRNAs and miRNAs with Associated ceRNA Networks in Triple-Negative Breast Cancer
}

\author{
Rui Yang ${ }^{a}$ Lei Xing ${ }^{b}$ Min Wang ${ }^{a}$ Hong Chia Luyu Zhang ${ }^{c}$ \\ Junxia Chen ${ }^{a}$ \\ aDepartment of Cell Biology and Genetics, Chongqing Medical University, Chongqing, bepartment \\ of Endocrine and breast surgery, The First Affiliated Hospital of Chongqing Medical University, \\ Chongqing, 'Molecular Medicine and Cancer Research Center, Chongqing Medical University, \\ Chongqing, China
}

\section{Key Words}

IncRNA • miRNA • ceRNA • Triple-negative breast cancer • Expression profiles

\begin{abstract}
Background/Aims: Triple-negative breast cancer (TNBC) is a subtype of highly malignant breast cancer with poor prognosis. Growing evidence indicates that Long noncoding RNAs (IncRNAs) play important regulatory roles in the development and progression of a variety of cancers including breast cancer. However, the underlying mechanisms remain largely unknown. Methods: Here, we compared the expression profiles of mRNAs, IncRNAs and miRNAs between 111 TNBC tissues and 104 non-cancerous tissues utilizing RNA-Seq Data from The Cancer Genome Atlas (TCGA). Gene Ontology and KEGG pathway enrichment analyses were executed to investigate the principal functions of the significantly dysregulated mRNAs. Moreover, Kaplan-Meier survival analyses were performed to determine the effects of differentially expressed IncRNAs/mRNAs/miRNAs on overall survival. Subsequently, we constructed a competing endogenous RNA (ceRNA) network, which included 66 dysregulated IncRNAs, 24 miRNAs and 55 mRNAs. The four dysregulated IncRNAs, three aberrantly expressed miRNAs and four mRNAs were confirmed in the ceRNA network by qRT-PCR in 30 pairs of samples, respectively. Results: A total of 1441 IncRNAs, 114 miRNA and 2501 mRNAs were found to be differentially expressed in TNBC tissues compared with controls. 109 IncRNAs and 124 mRNAs might serve as prognostic signature for patients with TNBC according to the survival analysis. Functional analysis revealed that 19 mRNAs in the ceRNA network were enriched in 17 cancerrelated pathways. Conclusion: Taken together, we identified novel IncRNAs/miRNAs which may serve as potential biomarkers to predict the survival and therapeutic targets for TNBC
\end{abstract}


Yang et al.: Analysis of Expression Profile of Lncrnas with Cerna in TNBC

patients based on a large-scale sample. More importantly, we constructed the ceRNA network of TNBC, which provides valuable information to further explore the molecular mechanism underlying tumorigenesis and development of TNBC.

(C) 2018 The Author(s)

Published by S. Karger AG, Basel

\section{Introduction}

Breast cancer is the most common malignant tumor in women and the leading cause of cancer-related death among women worldwide. Triple-negative breast cancer (TNBC), a subtype of breast cancer that is characterized by lack of expression of estrogen receptor (ER), progesterone receptor (PR) and human epidermal growth factor receptor 2 (HER2), accounting for about $15 \%$ of all breast cancers. The breast cancer subtype tends to be more frequent in younger patients ( $<50$ years old) with larger tumor size. TNBC is associated with aggressive and metastatic clinical behavior, early recurrence and poor prognosis. To date, no effective targeted therapies are available to treat TNBC [1]. Therefore, it is imperative to identify the potential biomarkers and novel therapeutic targets for a more precise recurrence prediction and therapy of TNBC.

Long non-coding RNAs (LncRNAs) are defined as transcripts longer than 200 nucleotides without evident protein coding function. LncRNAs constitute a heterogeneous class of RNAs, including sense, antisense, bidirectional, intronic and intergenic lncRNAs [2]. As the largest class of non-coding RNAs, the majority of the IncRNAs have not yet been functionally characterized to date. However, there is growing evidence indicating that lncRNAs may play crucial roles in regulating gene expression at transcriptional, post-transcriptional and epigenetic levels [3]. Recently, an increasing number of studies have demonstrated that lncRNAs participate in a wide variety of biological processes including genomic imprinting, transcription, chromosome modification, cell cycle, cell differentiation, invasion and migration, etc [4]. The aberrantly expressed lncRNAs have been found to be implicated in tumorigenesis and development of various cancers, including breast cancer [5]. Some wellstudied IncRNAs, such as H19, MALAT1 and HOTAIR have been reported to play oncogenic roles in the pathogenesis of tumors [6-8], whereas MEG3, GAS5 and BANCR could function as tumor suppressors [9-11]. However, the molecular mechanisms of lncRNAs underlying carcinogenesis and progression of triple-negative breast cancer remain largely unknown.

It is well known that microRNAs are a class of small noncoding RNAs with a length of 17-24 nucleotides and could regulate gene expression at post-transcriptional level via inhibiting protein translation or degrading target mRNAs. Emerging evidence shows that specific miRNAs may play an important role in progression and function as tumor suppressor genes or oncogenes [12]. Recently, Pandolfi et al. put forward competing endogenous RNA (ceRNA) hypothesis that mRNAs, non-coding RNAs and pseudogene transcripts can regulate each other through competing for the same microRNA response elements (MREs), which revealed a new mechanism of interaction among RNA transcripts [13]. Accumulating data show that IncRNAs as ceRNA are implicated in the tumorigenesis and progression of gastric cancer, colorectal cancer and so on $[14,15]$. However, up till now, little research has been done on ceRNA of TNBC.

The Cancer Genome Atlas (TCGA), a publically available database, is an abundant and valuable online resource for data mining and biological discovery, which contains more than 10,000 patient samples across 36 types of cancer up to now. The main goal of this study was to explore the molecular mechanisms underlying the non-coding RNAs in carcinogenesis and progression of TNBC and discover specific lncRNA markers in a large sample. Here, we conducted a comprehensive analysis of mRNA, IncRNA and miRNA expression profiles and predicted the possible biological functions of representative lncRNAs and clinical outcomes in a large cohort of patients with TNBC in TCGA database. More importantly, we first constructed a IncRNA-miRNA-mRNA ceRNA network in TNBC and the significant differential expressions of representative lncRNAs, miRNAs and mRNAs were further confirmed with qRT-RCR. Furthermore, we identified a specific lncRNA signature associated with prognosis,

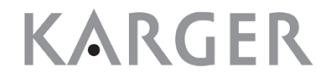




\section{Cellular Physiology Cell Physiol Biochem 2018;50:473-488 \begin{tabular}{l|l} 
DOI: 10.1159/000494162 & $\begin{array}{l}\text { O } 2018 \text { The Author(s). Published by S. Karger AG, Basel } \\
\text { www.karger.com/cpb }\end{array}$
\end{tabular}}

Yang et al.: Analysis of Expression Profile of Lncrnas with Cerna in TNBC

tumorigenesis and development of TNBC. Our data reveal the mechanisms underlying tumorigenesis and progression of TNBC and provide novel diagnostic or prognostic markers and therapeutic targets for TNBC.

\section{Materials and Methods}

\section{Patients and samples}

This study was approved by the Ethics Committee of Chongqing Medical University and conducted in accordance with the Declaration of Helsinki. A total of 1097 patients with breast invasive carcinoma and corresponding clinical information were obtained from TCGA data portal (https://tcga-data.nci.nih.gov/ tcga/). The exclusion criteria were as follows: 1) histologic diagnosis was not TNBC; 2) suffering from other malignancies; 3) patients had received preoperative chemoradiation; and 4) patients without complete data for analysis. As a result, 111 patients with TNBC were included in our study. The RNA expression profiles of control group were obtained from adjacent non-tumor breast tissues $(n=104)$. The study was completely in conformity with the publication guidelines provided by TCGA (https://cancergenome.nih.gov/publications/ publicationguidelines). In addition, 30 pairs of TNBC tissues and para-cancerous tissues were collected from patients, who did not suffer from other malignancies or receive preoperative chemotherapy or radiotherapy, in The First Affiliated Hospital of Chongqing Medical University. Informed consents were obtained from all patients. Tissues were snap-frozen in liquid nitrogen immediately after surgical resection and stored until RNA extraction.

Acquisition and analysis of $m R N A$, IncRNA and miRNA expression profiles

The RNAseq and miRNAseq data (level 3) of the corresponding samples were downloaded from TCGA data portal. The IncRNA and mRNA expression profiles were generated from Illumina HiSeq 2000 RNA Sequencing platform and the miRNA expression profiles were produced by Illumina HiSeq 2000 miRNA Sequencing platform. As these expression data have already been normalized by TCGA, no further normalization was needed.

After deletion of the data with no expression, the level 3 RNAseq and miRNAseq data between tumor tissues and non-tumor tissues were analyzed by edgeR which is a Bioconductor package based on R language for differential expression analysis of RNAseq data (http://www.bioconductor.org/packages/release/ bioc/html/edgeR.html). Genes with FDR $<1 \%$, absolute log 2 fold change $>2$ and $\mathrm{P}<0.01$ were regarded as differentially expressed genes and sent for further analysis. In our study, only lncRNAs with description from Ensembl were extracted for further study, while the ones with no annotation were excluded.

\section{RNA extraction}

Total RNA was extracted from TNBC tissues and adjacent non-cancerous tissues using TRIzol reagent (TAKARA, Dalian, China) according to manufacturer's protocol. The quantity and quality of RNA were evaluated by Nano Drop ND-1000 spectrophotometer (Nano Drop Thermo, Wilmington, DE, USA), and the integrity of RNA was detected by agarose gel electrophoresis.

\section{qRT-PCR validation for representative RNAs}

30 pairs of TNBC tissues samples and adjacent non-cancerous tissues were used for qRT-PCR validation. After RNA isolation, M-MLV reverse transcriptase (Invitrogen, USA) was used for synthesizing cDNA according to the manufacturer's instructions. Subsequently, we performed qRT-PCR using SYBR Green assays in a total reaction volume of $10 \mu \mathrm{l}$, including $0.5 \mu \mathrm{l}$ PCR Forward Primer $(10 \mu \mathrm{M}), 0.5 \mu \mathrm{l}$ PCR Reverse Primer $(10 \mu \mathrm{M}), 2 \mu \mathrm{l}$ cDNA, $5 \mu \mathrm{l} 2 \times$ Master Mix and $2 \mu \mathrm{l}$ double distilled water. The protocol was initiated at $95^{\circ} \mathrm{C}$ for $10 \mathrm{~min}$, then at $95^{\circ} \mathrm{C}(10 \mathrm{sec}), 60^{\circ} \mathrm{C}(60 \mathrm{sec})$ for a total $40 \mathrm{cycles}$. $\beta$-actin and U6 were used as internal references for quantification of IncRNAs, mRNAs and miRNAs, respectively. Results were obtained from three independent wells. For quantitative results, the relative expression level of each IncRNA, miRNA and mRNA was calculated using $2^{-\Delta \Delta C t}$ method. The values were expressed as means $\pm \mathrm{SD}$. The primers are below. 


\title{
Cellular Physiology Cell Physiol Biochem 2018;50:473-488 \begin{tabular}{l|l|l} 
and Biochemistry 10.1159/000494162 & $\begin{array}{l}\text { C } 2018 \text { The Author(s). Published by S. Karger AG, Basel } \\
\text { www.karger.com/cpb }\end{array}$
\end{tabular}
}

Yang et al.: Analysis of Expression Profile of Lncrnas with Cerna in TNBC

\begin{abstract}
LINC00452: F, 5'- TCTGGCTAGGAAGCAAGCAC-3'; R, 5'- TGTATGGCTGTGGATCTGCG-3'. ST7-AS2: F, 5'- GGCAAGGTCAGAGAGATCTGG-3'; R, 5'-TGAGTGGTGGGTATGACCAGT-3'. LINC00461: F, 5' - TGGAGAAGCCTGTTAAACCACT-3'; R, 5' - ACAAGGGTCTTCTCAACGGTC-3'. LINC00517: F, 5'- AATCTGGGACCCCTGTGAGTA-3'; R, 5' - ATAAGTTCCTGCGCTGTCCTG-3'. hsa-miR-145: F, 5'-ATGGTTCGTGGGGTCCAGTTTTCCCAGGAATC-3'; R, 5'-GTGCAGGGTCCGAGGT-3'; RT, GTCGTATCCAGTGCAGGGTCCGAGGTATTCGCACTGGATACGACCAGGGATTC. hsa-miR-144: F, 5'-ATGGTTCGTGGGTACAGTATAGATGATGTACT-3'; R, 5'-GTGCAGGGTCCGAGGT-3'; RT, GTCGTATCCAGTGCAGGGTCCGAGGTATTCGCACTGGATACGACCAGTACATC. hsa-miR-137: F, 5'-ATGGTTCGTGGGTTATTGCTTAAGAATACGCG-3'; R, 5'-GTGCAGGGTCCGAGGT-3'; RT, GTCGTATCCAGTGCAGGGTCCGAGGTATTCGCACTGGATACGACCCTACGCGT. EZH2: F, 5'- AGTTCGTGCCCTTGTGTGAT-3'; R, 5'- TGTTGCACTGTGCTTTGCAG-3'. FSCN1: F, 5' - AGGACGAGCTCTTTGCTCTG-3'; R, 5'- CGGTCTCCTCGTCCTGATTG-3'. KLF4: F, 5'- AATTCGCTGACCCATCCTCC-3'; R, 5' - CCGGATCGGATAGGTGAAGC-3'. SOX11: F, 5' - TGCCAGTGCACAGATAGGAC-3'; R, 5'- ACCACATCTCAACTCGGTCA-3'. $\beta$-actin: F, 5'-CATGTACGTTGCTATCCAGGC-3'; R, 5'-CTCCTTAATGTCACGCACGAT-3'. U6:F,5'-CTCGCTTCGGCAGCACA-3';R,5'-AACGCTTCACGAATTTGCGT-3';RT, AACGCTTCACGAATTTGCGT.
\end{abstract}

Construction of ceRNA network

The IncRNA-miRNA-mRNA ceRNA network was based on the ceRNA hypothesis that ceRNAs could co-regulate each other by competing for shared microRNA response elements (MR Es). To construct the network, IncRNA-miRNA interactions were retrieved from miRcode (http://www.mircode.org/), miRTarBase (http://mirtarbase.mbc.nctu.edu.tw/) and Targetscan (http://www.targetscan.org/) were used to predict the target genes of miRNAs. Then, differentially expressed IncRNA, miRNA and mRNA with FDR $<1 \%$, absolute log 2 fold change $>2$ and $\mathrm{P}<0.01$ were retained to establish the ceRNA network. Cytoscape 3.4 .0 was applied to construct and visualize the network.

\section{Functional enrichment analysis}

Gene Ontology (GO) and Kyoto Encyclopedia of Genes and Genomes (KEGG) pathway enrichment analyses were performed to predict the functions of differentially expressed mRNAs and mRNAs involved in the ceRNA network with the DAVID Bioinformatics Tool (https://david.ncifcrf.gov/,version 6.8) and KOBAS 3.0 (http://kobas.cbi.pku.edu.cn/index.php), respectively. The results of GO analysis have covered domains including biological process, cellular component and molecular function. GO terms and KEGG pathways with a P value of $<0.01$ and an enrichment score of $>2.0$ were considered as significantly enriched function annotations.

\section{Statistical analysis}

Kaplan-Meier survival analyses were utilized to assess the overall survival of TNBC patients who were classified as high expression and low expression group based on the median expression level of each differentially expressed RNA. And the differences in patients' survival were estimated by log-rank test with the R package "survival". Student's t-test was used to compare the difference in two groups. P value less than 0.05 was considered as statistically significant. The statistical analyses were performed using SPSS 19.0 (Chicago, IL).

\section{Results}

Differentially expressed mRNAs (DEmRNAs) and annotation for function in TNBC

We performed differential expression analysis by comparing the RNA expression levels in 111 TNBC tissues and 104 normal tissues. The detailed clinical and pathological characteristics of the patients in the study were listed in Table 1. A total of 2501 DEmRNAs were identified with a FDR $<1 \%$, an absolute value of log 2 fold change $>2$ and $\mathrm{P}<0.01$, among which 1583 mRNAs were up-regulated and 918 mRNAs were down-regulated. The top 100 up-regulated and top 100 down-regulated mRNAs were outlined in Fig. S1 (for all supplementary material see www.karger.com/10.1159/000494162/). The top 5 


\section{Cellular Physiology Cell Physiol Biochem 2018;50:473-488 \begin{tabular}{l|l} 
DOI: 10.1159/000494162 & $\begin{array}{l}\text { O } 2018 \text { The Author(s). Published by S. Karger AG, Basel } \\
\text { www.karger.com/cpb }\end{array}$
\end{tabular}}

Yang et al.: Analysis of Expression Profile of Lncrnas with Cerna in TNBC

up-regulated mRNAs were FTHL17, CSAG1, ACTL8, MAGEA12 and MAGEA3. Meanwhile, the top 5 down-regulated mRNAs were MYH2, MYL1, XIRP2, CSRP3 and MYL2. Heat map for the hierarchical clustering of the differentially expressed mRNAs was carried out by the pheatmap function of the R language package.

In order to understand the potential functions of the dysregulated mRNAs that may be involved in the tumorigenesis and development of TNBC, we performed Gene Ontology (GO) and KEGG pathway enrichment analysis using DAVID and KOBAS 3.0, respectively. Our analysis revealed the top 30 significantly enriched GO terms and top 10 enriched KEGG pathways of the DEmRNAs (Fig. S2). The enriched GO terms were mainly associated with DNA replication, cell differentiation and proliferation, cell division, cell adhesion and cellular protein metabolic process. While, the DEmRNAs were significantly enriched in cancerrelated pathways, including metabolic pathways, PI3K-Akt signaling pathway, pathway in cancer, transcriptional misregulation in cancer, cell cycle, proteoglycans in cancer and PPAR signaling pathway. The results of the functional enrichment analyses suggested that these DEmRNAs could participate in tumorigenesis and development of TNBC through regulating related biological processes and key pathways.

To investigate the relationship between the DEmRNAs and prognostic characteristics, we conducted Kaplan-Meier curve analysis for the 2501 DEmRNAs in the patients with TNBC. Consequently, 124 DEmRNAs were significantly associated with patients' overall survival, among which 48 DEmRNAs were negatively associated, while 76 DEmRNAs were positively associated with the prognosis of patients, respectively (Table S1). As shown in Fig. 1, LHX1, WISP1 and S1PR1 were negatively associated, while SORBS1 was positively correlated with patients' overall survival.

Differentially expressed IncRNAs (DEInRNAs) and analysis associated with survival in TNBC

A total of 1441 lncRNAs were identified as being differentially expressed in TNBC tissues compared to the normal tissues with a FDR $<1 \%$, an absolute value of $\log 2$ fold change $>2$ and $\mathrm{P}<0.01$. The expressions of 1039 lncRNAs were significantly up-regulated and 402 lncRNAs were remarkably down-regulated. Among them, the top 100 up-regulated and top 100 downregulated IncRNAs were depicted in Fig. 2. The top 5 up-regulated lncRNAs were RP1154H7.4, LINC00392, RP11-366F6.2, RP11-77A13.1 and TLX1NB. Whereas the top 5 downregulated lncRNAs were MIR1-1HG, RP11-451G4.2, RP11-133L19.3, C10orf71-AS1 and RP11-798K3.2. The result of bidirectional hierarchicshal clustering showed distinguishable lncRNA expression patterns among samples. All of the 1441 lncRNAs were found to be involved in the pathogenesis and development of TNBC. Then, Kaplan-Meier curve analysis was performed to investigate overall survival for the 1441 DElncRNAs in patients with TNBC. We found that the expressions of 75 DElncRNAs were negatively associated and 34 DElncRNAs were positively associated with patients' overall survival, respectively (Table S2). Representative results for survival analyses were shown in Fig. 3. C8orf31 and LINC00452 were negatively related with patients' overall survival, whereas RP11-3J1.1 and RP11-496D24.2 were positively associated with prognosis of patients.

Table 1. Clinicopathological characteristics of 111 TNBC patients

\begin{tabular}{lcc}
\hline Parameter & Subtype & Patient n(\%) \\
\hline Vital status & alive & $95(85.59)$ \\
& dead & $16(14.41)$ \\
Age(years) & $<68$ & $96(86.49)$ \\
& $>=68$ & $15(13.51)$ \\
Gender & Female & $111(100)$ \\
Race & Asian & $8(7.21)$ \\
& Black or African American & $32(28.83)$ \\
& unknow & $6(5.41)$ \\
Pathologic stage & White & $65(58.56)$ \\
& Stage I & $17(15.32)$ \\
& Stage II & $72(63.96)$ \\
Pathologic T & Stage III & $20(16.22)$ \\
& Stage IV & $2(1.8)$ \\
& T1 & $22(19.82)$ \\
Pathologic N & T2 & $76(68.47)$ \\
& T3 & $12(10.81)$ \\
& T4 & $1(0.90)$ \\
& N0 & $71(63.96)$ \\
Pathologic M & N1 & $25(22.52)$ \\
& N2 & $11(9.91)$ \\
& N3 & $4(3.60)$ \\
& M0 & $94(84.68)$ \\
& M1 & $2(1.80)$ \\
& MX & $15(13.51)$ \\
\hline
\end{tabular}


Fig. 1. KaplanMeier curveanalysis of DEmRNAs for the overall survival in TNBC patients. Four representative $\mathrm{D} \mathrm{E} \mathrm{m} \mathrm{R} \mathrm{N} \mathrm{s}$, including LHX1, WISP1, S1PR1 and SORBS1 were showed $\quad(p<0.05)$. Horizontal axis: overall survival time, years; Vertical axis: overall survival rate.


Fig. 2. Heat map with bidirectional hierarchical clustering of D E ln c R N A s in TNBC. The top 100 upregulated and 100 down$\mathrm{regulated}$ D E ln c R N A s were displayed by heat map. Each column represents a sample and each row repres ents a transcript. The red strip repres ents high relative expression and the green strip repres en t s

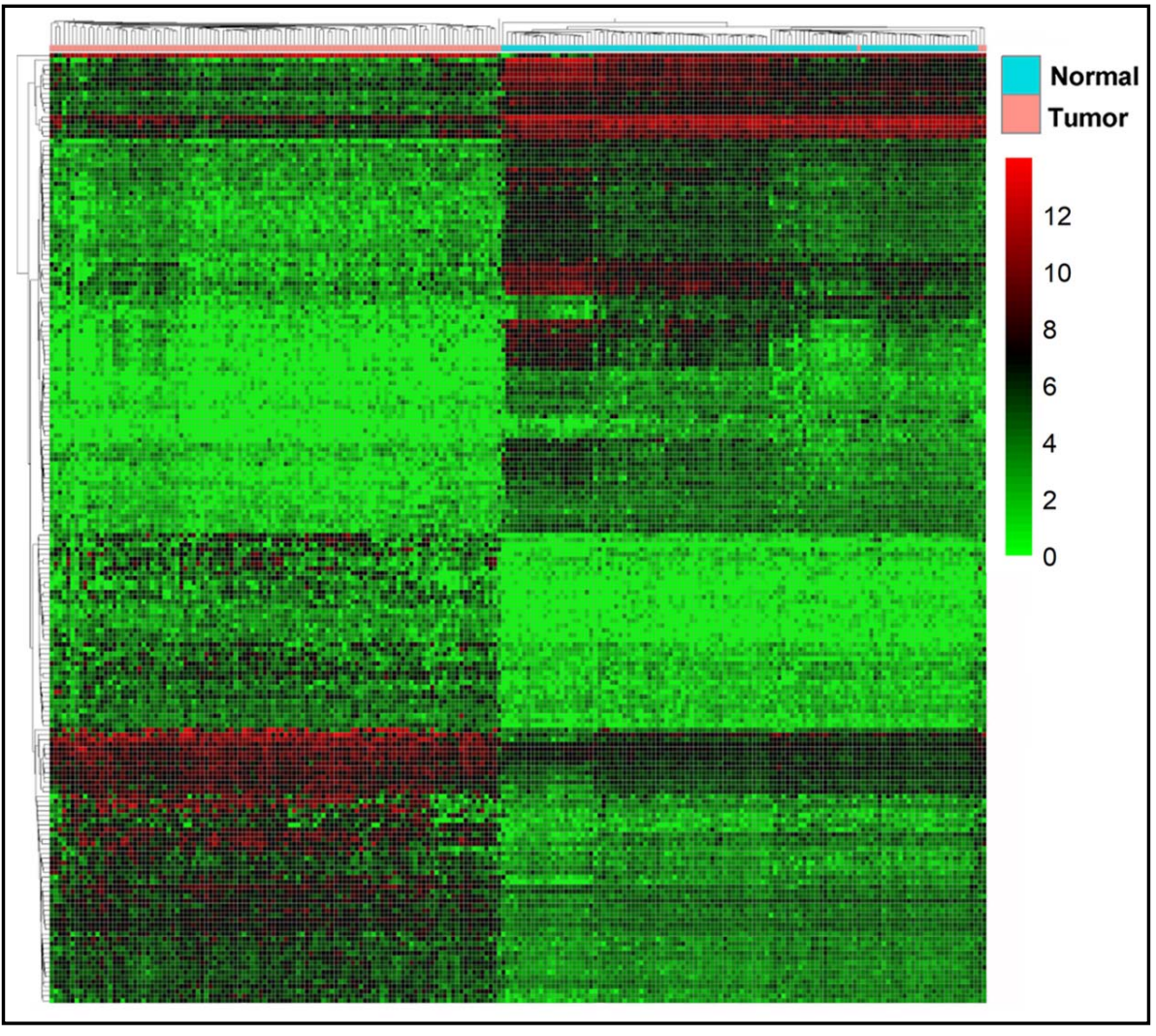
low relative expression. 
Fig. 3. Kaplan-Meier curve analysis of DElncRNAs for the overall survival in TNBC patients. Four representative D E l n C R N A , including C8orf31, LINC00452, RP113J1.1 and RP11496D24.2 were exhibited $(\mathrm{p}<0.05)$. Horizontal axis: overall survival time, years; Vertical axis: overall survival rate.

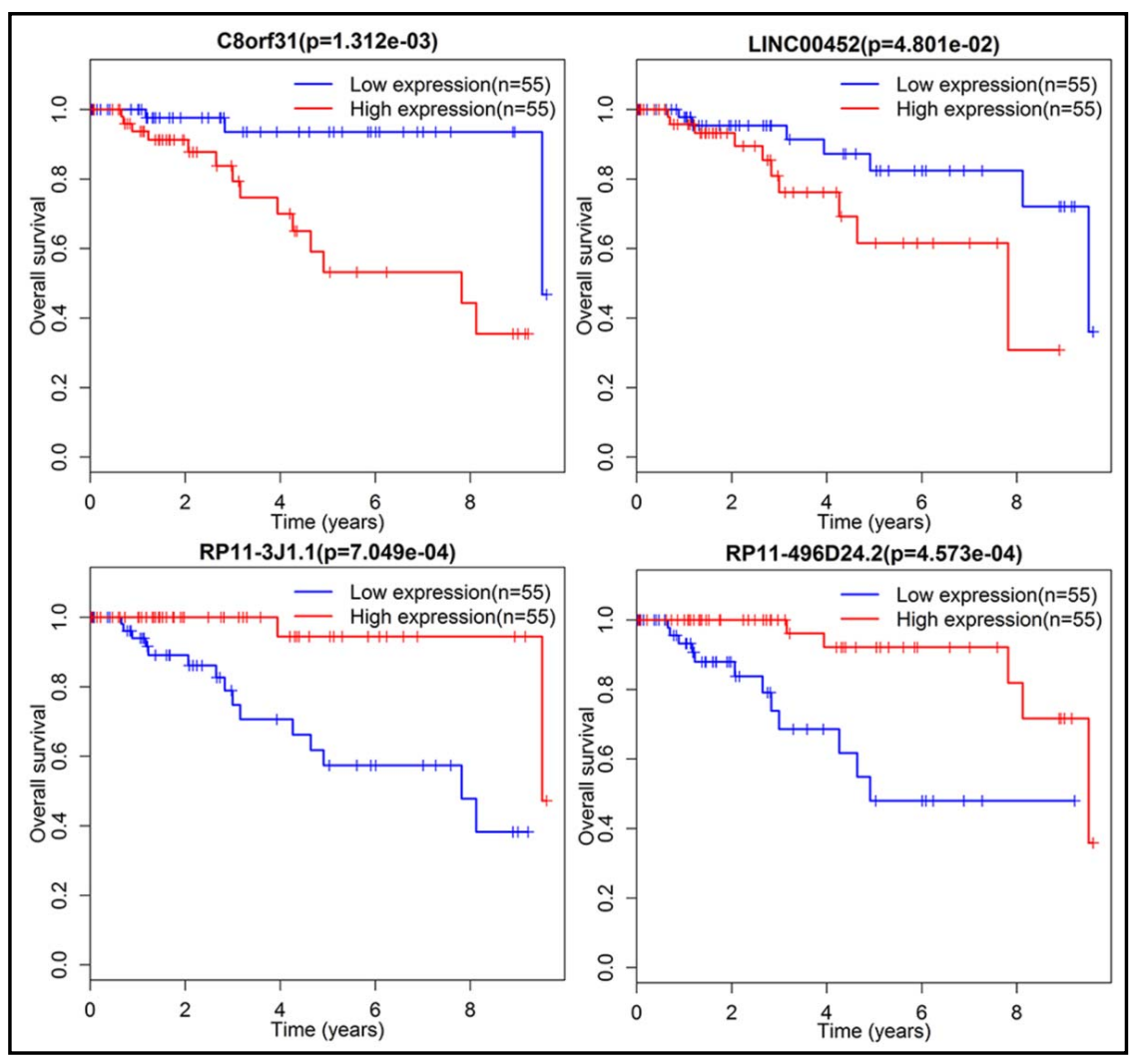

Differentially expressed miRNAs (DEmiRNAs) and analysis associated with survival in $T N B C$

In order to probe IncRNA-miRNA-mRNA interactions in TNBC, we investigated miRNA expression profiles in tumor tissues and normal tissues. Using the same cut-off criteria as mRNA and lncRNA, a total of 114 DEmiRNAs were identified, including 91 up-regulated and 23 down-regulated. The top 5 up-regulated miRNAs were hsa-miR-105-2, hsa-miR-105-1, hsa-miR-1269a, hsa-miR-767 and hsa-miR-1269b, while hsa-miR-133a-2, hsa-miR-133a-1, hsa-miR-1-1, hsa-miR-1-2 and hsa-miR-133b were identified as the top 5 down-regulated miRNAs. Hierarchical clustering was exhibited in Fig. S3. Kaplan-Meier curve analysis was also carried out to investigate overall survival for DEmiRNAs in TNBC patients. Representative survival curves were shown in Fig. 4. The expressions of hsa-miR-216a and hsa-miR-135b were positively correlated with overall survival of the patients, while the levels of hsa-miR-7706 and hsa-miR-3610 were significantly negatively correlated with overall survival of the patients (Table S3).

\section{Construction of ceRNA network and prediction of function in TNBC}

The ceRNA hypothesis suggests that competing endogenous RNAs (ceRNAs) could competitively bind to the microRNA response elements (MREs) to communicate with and co-regulate each other. A ceRNA network was constructed according to above data (Fig. 5A). In the network, we selected 66 DElncRNAs that could bind to 24 DEmiRNAs based on miRcode. Then, mRNAs targeted by the DEmiRNAs were searched using two target gene prediction databases miRTarBase and Targetscan. As a result, 55 DEmRNAs were found in the ceRNA network. Noticeably, the IncRNA LINC00461 could interact with 9 DEmiRNAs, including has-miR-139, has-miR-455, has-miR-141, has-miR-144, has-miR-200a, has-miR106a, has-miR-137, has-miR-211 and has-miR-216a. To better understand the functions of DElncRNAs in the ceRNA network, we performed KEGG pathway analysis of the DEmRNAs of the ceRNA network. A total of 34 significantly enriched pathways were found, the top 20 
Fig. 4. KaplanMeier curveanalysis of DEmiRNAs for the overall survival in TNBC patients. Four DEmiRNAs, including hsamiR-135b, hsamiR-216a, hsamiR-3610 and hsamiR-7706, were presented $(\mathrm{p}<0.05)$. Horizontal axis: overall survival time, years; Vertical axis: overall survival rate.
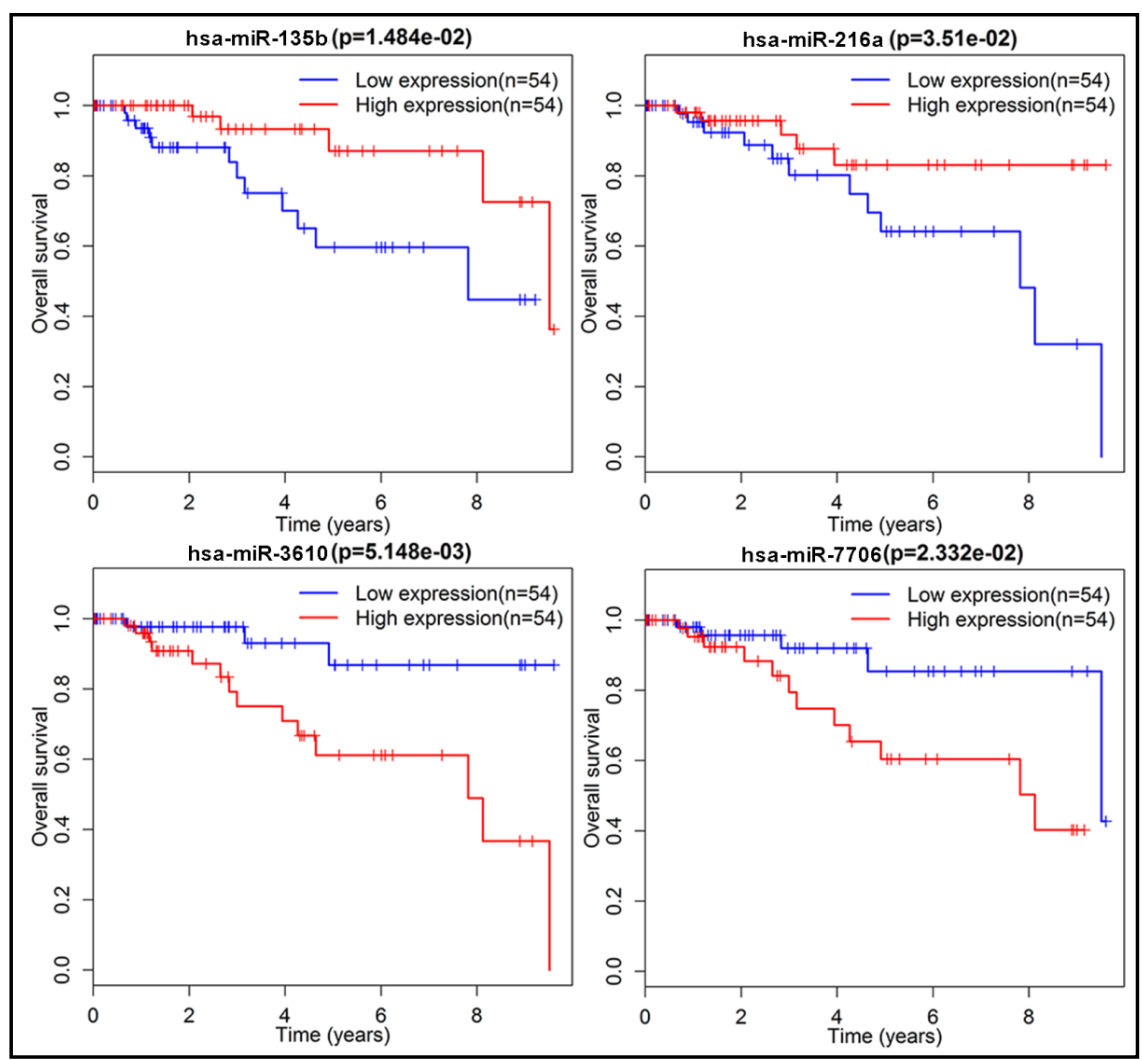

enriched pathways were shown in Fig. S4. Some of which were cancer-related pathways such as microRNAs in cancer, melanoma, proteoglycans in cancer, apoptosis, calcium signaling pathway, transcriptional misregulation in cancer, pathways in cancer, colorectal cancer, rap1 signaling pathway, colorectal cancer, pancreatic cancer, Ras signaling pathway, chronic myeloid leukemia, MAPK signaling pathway, cytokine-cytokine receptor interaction, prostate cancer and choline metabolism in cancer. While other non-cancer related pathways were also enriched, including signaling pathways regulating pluripotency of stem cells, endocrine resistance, estrogen signaling pathway and so on.

The ceRNA network revealed that these DElncRNAs might function as ceRNAs to regulate or interact with mRNAs via miRNAs in TNBC. To validate the speculation, the regression analyses were executed between the expression levels of the representative DElncRNA and DEmRNAs in the network. The positive correlations were found between the expression levels of IncRNA LINC00461 and mRNAs ELAVL2, ZNF367, EZH2 or MYCN, suggesting LINC00461 could competitively bind to miR-141, miR-139 or miR-144 through microRNA response elements (MREs) to relieve microRNA-mediated repression of target genes (Fig. 5B).

Validation of representative dysregulated IncRNAs, miRNAs and mRNAs in TNBC

Four IncRNAs, three miRNAs and four mRNAs from the ceRNA network were chose for verification of TCGA data in 30 pairs of samples by quantitative real-time PCR. The qRTPCR assay showed that the expressions of IncRNA LINC00452, ST7-AS2 and LINC00461 were upregulated, while LINC00517 was downregulated in TNBC compared with control group, respectively. Meanwhile, the expressions of hsa-miR-145 and hsa-miR-144 were downregulated, hsa-miR-137 was upregulated, and the expressions of 3 target mRNAs EZH2, FSCN1 and SOX11 were upregulated, whereas KLF4 was downregulated in TNBC compared with control group (Fig. 6). The results were consistent with data from TCGA. More importantly, we found that IncRNA LINC00461 and mRNA EZH2 displayed high co- 


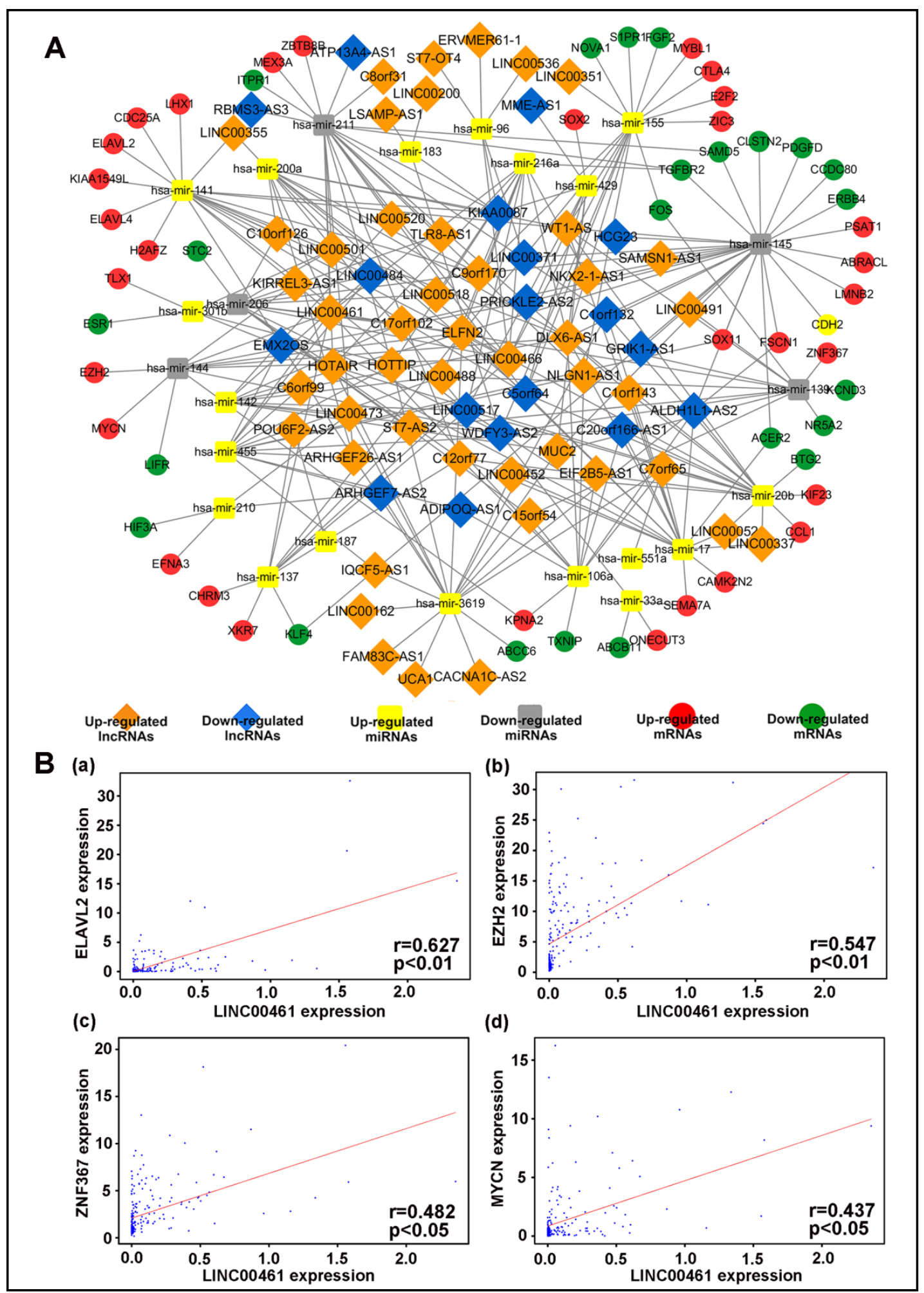

Fig. 5. LncRNA-miRNA-mRNA ceRNA network in TNBC. (A) Overview of the lncRNA-miRNA-mRNA ceRNA network in TNBC. Orange diamonds represent up-regulated lncRNAs, yellow round rectangles represent up-regulated miRNAs and red balls represent up-regulated mRNAs. Blue diamonds represent downregulated lncRNAs, gray round rectangles represent down-regulated miRNAs and green balls represent down-regulated mRNAs. (B) Correlation between expression levels of ceRNAs. (a) LINC00461 vs. ELAVL2. (b) LINC00461 vs. EZH2. (c) LINC00461 vs. ZNF367. (d) LINC00461 vs. MYCN. r: correlation coefficient. 
Fig. 6. Validation of the expression of representative dysregulated mRNAs, IncRNAs and miRNAs by quantitative RTPCR in TNBC. The relative expression of four lncRNAs, three miRNAs and mRNAs was showed between tumor tissues (T) and adjacent noncancerous tissues (N). Data were presented as mean $\pm S D, n=30$.
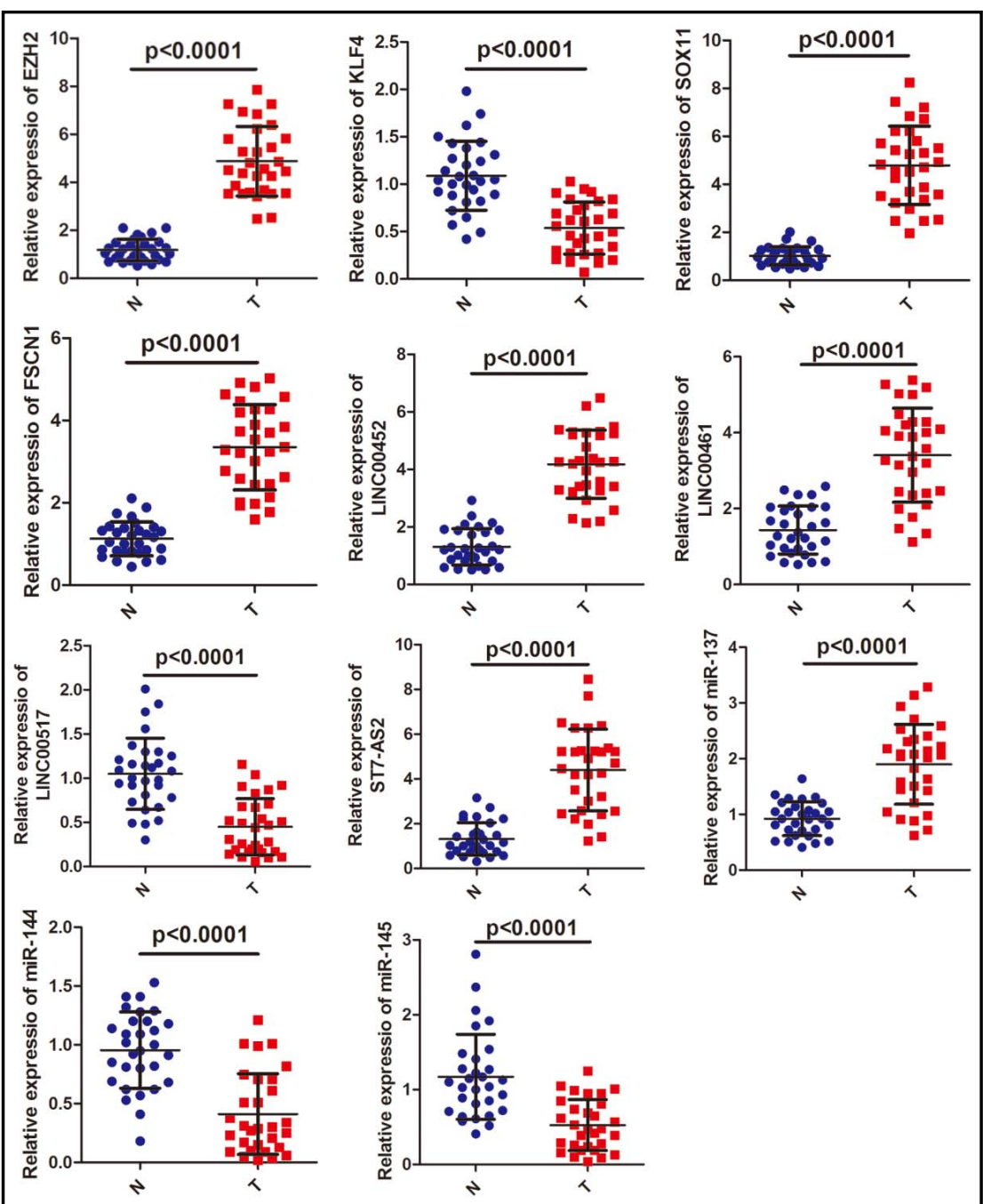

Table 2. Correlations between pathological grade and DElncRNAs from the ceRNA network

\begin{tabular}{|c|c|}
\hline Down-regulated & Up-regulated \\
\hline Pathologic_T $(\mathrm{T} 3+\mathrm{T} 4$ vs. $\mathrm{T} 1+\mathrm{T} 2)$ & $\begin{array}{l}\text { ST7-AS2, ST7-OT4, NLGN1-AS1, HOTTIP, LINC00491, } \\
\text { C1orf143 }\end{array}$ \\
\hline Pathologic_N（N2+N3 vs. N0+N1） & C10orf126 \\
\hline Pathologic_M（M1 vs. M0 ） & ST7-AS2, ST7-OT4, NLGN1-AS1, C8orf31, ELFN2 \\
\hline Pathologic_Stage（StageI+II vs. StageIII+IV ) & C10orf126 \\
\hline
\end{tabular}

expression; while IncRNA LINC00517 and mRNA KLF4 were consistently down-regulated in TNBC. IncRNA LINC00461 or IncRNA LINC00517 expression was negatively correlated with the level of hsa-miR-144 or hsa-miR-137 in TNBC. Our data suggest that these RNA transcripts could mutually regulate each other through competing for their shared miRNAs and might be implicated in the pathogenesis and development of TNBC.

\section{Correlations between pathological grade and DEIncRNAs from the ceRNA network}

To further explore whether DElncRNAs in the ceRNA network were implicated in the development and progression of TNBC, tumor tissue samples were assigned to several subgroups according to pathological tumor-node-metastasis (TNM) classification (T3+T4 vs. $\mathrm{T} 1+\mathrm{T} 2, \mathrm{~N} 2+\mathrm{N} 3$ vs. $\mathrm{N} 0+\mathrm{N} 1, \mathrm{M} 1$ vs. M0, stage I-II vs. stage III-Iv). We analyzed the expression levels of the lncRNAs in each subgroup. The results found that 9 lncRNAs were 
significantly differentially expressed in TNBC and associated with the TNM staging system. As demonstrated in Table 2, six lncRNAs (ST7-AS2, ST7-OT4, NLGN1-AS1, HOTTIP, LINC00491 and C1orf143) were dysregulated in pathological T stage, one lncRNA (C1orf143) was significantly differentially expressed in pathological N stage, five lncRNAs (ST7-AS2, ST7-OT4, NLGN1-AS1, C8orf31 and ELFN2) were remarkably differentially expressed in pathological M stage. Moreover, the expression of IncRNA C10orf126 was positively correlated with pathological grade of TNBC. And all of them were up-regulated. In addition, our analysis also found that four lncRNAs RBMS3-AS3, C8orf31, LINC00452 and LINC00200 could be used as prognostic predictors in TNBC. Our findings suggest that these DElncRNAs could contribute to the development and progression of TNBC and might have substantial clinical significance for the targeted therapy of TNBC in the future.

\section{Discussion}

Women with TNBC have a higher rate of distant recurrence and a poorer prognosis than patients with other breast cancer subtypes. Due to the heterogeneity of the disease and lack of well-defined therapeutic targets, the treatment of TNBC has been a major challenge. Thus, specific targets and predictors would be of great value in the diagnosis and therapy of TNBC [16]. Accumulating evidence indicates that aberrantly expressed lncRNAs play crucial roles in tumorigenesis and progression by functioning as tumor suppressors or oncogenes. Compared with protein coding gene, IncRNAs are considered to have a high tissue- and disease-specific expression patterns, moreover, their expressions are more closely related to tumor grade and stage [17]. In recent years, some dysregulated lncRNAs in various cancers including gastric cancer, lung cancer, breast cancer, etc., have been discovered by transcriptional profile analysis and experimental researches, highlighting their potential roles as novel biomarkers and therapeutic targets for cancers [18-20]. However, genomewide screen of the expression of IncRNAs and validation to elucidate the functions of specific lncRNAs and clinical implications by integrated miRNA and mRNA expression profiles in TNBC based on large-scale RNA-seq data has not been reported so far.

In the present study, we integrated IncRNA/mRNA/miRNA expression profiles to perform a comprehensive analysis for the IncRNA functions and related prognosis in a large cohort of TNBC patients based on the data derived from TCGA database. A total of 1441 differentially expressed IncRNAs were identified, among which 1039 lncRNAs were significantly up-regulated and 402 were remarkably down-regulated. Four dysregulated IncRNAs, three aberrantly expressed miRNAs and four mRNAs were randomly selected for verification of the TCGA data in 30 pairs of samples by qRT-PCR. The results of qRT-PCR were consistent with the data from TCGA. In addition, several well-studied lncRNAs were also found to be differentially expressed in our analysis. UCA1, for instance, has been reported by researchers to promote breast cancer growth through suppressing the expression of p27 with the interaction between UCA1 and hnRNP I [21]. But, our data did not found changes in p27 expression, suggesting UCA1 might have additional functions besides the interaction of UCA1 with hnRNP I. Our results showed that the HOTAIR and TINCR were significantly upregulated in TNBC. It is known that HOTAIR and TINCR have been implicated in breast tumorigenesis as oncogene. Xu et al. demonstrated that the knockdown of TINCR and HOTAIR inhibited breast cancer cell proliferation, induced apoptosis and inhibited cell cycle progression in vitro [22]. Xue et al. reported that HOTAIR was upregulated in tamoxifenresistant breast cancer tissues compared to their primary counterparts. HOTAIR was directly repressed by estrogen receptor and its upregulation promoted ligand-independent estrogen receptor activities and contributed to tamoxifen resistance [23]. Zhou et al. showed that HOTAIR efficiently enhanced radioresistance in MDA-MB231 breast cancer cells and accelerated proliferation through the Akt pathway by targeting HOXD10 [24]. In addition, some dysregulated IncRNAs in our investigation, such as HOTTIP, HOXA11-AS, BCAR4 and LINC00511, were also demonstrated by other researchers. Li et al. showed that HOXA11- 


\section{Cellular Physiology Cell Physiol Biochem 2018;50:473-488 \begin{tabular}{l|l|l} 
DOI: 10.1159/000494162 & $\begin{array}{l}\text { O 2018 The Author(s). Published by S. Karger AG, Basel } \\
\text { www.karger.com/cpb }\end{array}$
\end{tabular} \\ Yang et al.: Analysis of Expression Profile of Lncrnas with Cerna in TNBC}

AS expression in breast cancer tissue of 50 patients was relatively higher than that in adjacent normal tissues. HOXA11-AS promoted breast cancer invasion and metastasis by regulating epithelial-mesenchymal transition [25]. The expression of HOTTIP was found to be significantly correlated with tumor size, lymph node status, TNM stage and overall survival in the breast cancer patients [26]. Our data further support these findings. Next, we focused on the prognostic significance of the dysregulated IncRNAs. Kaplan-Meier survival curves revealed that 109 out of 1441 dysregulated lncRNAs were significantly associated with patients' overall survival. Importantly, four lncRNAs including RBMS3-AS3, C8orf31, LINC00452 and LINC00200 were not only found to be involved in the ceRNA network, but also were inversely associated with overall survival in TNBC patients, strongly suggesting that these lncRNAs could be implicated in tumorigenesis and development of TNBC and might be prospective clinical markers of diagnosis, therapy and prognosis for TNBC.

miRNAs are a class of non-coding RNA molecules that play important roles in regulating gene expression during normal or pathologic cellular process at the posttranscriptional level [27]. Genome-wide prediction suggests that more than $60 \%$ of protein coding genes can be regulated by miRNAs. The dysregulation of specific miRNAs could be associated with different cancers including TNBC [28]. Here, 114 differentially expressed miRNAs were identified in TNBC tissues compared with normal adjacent tissues, among which 91 miRNAs were up-regulated and 23 miRNAs were down-regulated. Our data showed that miR-210 was significantly up-regulated in TNBC. It has been reported that the microRNA miR-210 was highly expressed in TNBC, and its overexpression had been correlated with poor prognosis [29]. Lü.et al. showed that the expression of miR-210 was associated with shorter overall survival in TNBC [30]. By combining the miRNAseq data with clinical data obtained from TCGA database, we found that 4 miRNAs including hsa-miR-3610, hsa-miR-135b, hsamiR-7706 and hsa-miR-216a were significantly associated with patients' overall survival. Specifically, hsa-miR-216a was positively associated with patients' prognosis. The miR-216a has been reported to be a tumor suppressor in pancreatic cancer and colorectal cancer [31, 32], which is in line with our data. We found that miR-301b and miR-96 were significantly upregulated in TNBC compared with control. Koduru et al. showed that miR-301b was one of the top upregulated microRNAs and miR-96 was highly dysregulated along with miR-301b in TNBC [33]; While, miR-206 was substantially downregulated in TNBC. Wang et al. showed that miR-206 inhibited cell migration through direct targeting of the actin-binding protein coronin $1 \mathrm{C}$ in TNBC [34]. Our data are consistent with these researches, suggesting these microRNAs might be involved in the tumorigenesis and development of TNBC.

Furthermore, we also performed genome-wide mRNAs expression profiling in TNBC. As a result, we found that 2501 mRNAs were differentially expressed between TNBC tissues and non-tumor tissues according to TCGA data. Among them, 1583 and 918 mRNAs were upregulated and downregulated. Moreover, in line with previous study, our analysis revealed a remarkable upregulation of the checkpoint kinase 1 (CHEK1), which may be a therapeutic target in TNBC [35]. While, we found an obvious decrease of AP-1 protein called FOSB, the expression of which is negatively associated with the pathological grade of TNBC [36].

It was well known that lncRNAs could regulate the neighboring and overlapping coding gene expressions. Therefore, IncRNAs implement functions that might be embodied in related mRNA genes. Gene Ontology and KEGG pathway enrichment analyses of significantly differentially co-expressed mRNAs could provide insight into the role of dysregulated lncRNAs [37]. We found that these differentially expressed mRNAs were significantly enriched in 223 GO terms and 97 KEGG pathways. These significant GO terms are mainly involved in DNA replication, cell differentiation and proliferation, cell division, cell adhesion and cellular protein metabolic process. KEGG pathway analysis displayed that 7 cancer-related pathways were significantly enriched in metabolic pathways, PI3K-Akt signaling pathway, pathway in cancer, transcriptional misregulation in cancer, cell cycle, proteoglycans in cancer and PPAR signaling pathway, suggesting these mRNAs could play the crucial roles in the tumorigenesis and development of TNBC. Our data support Chen et al.'s finding [38]. Subsequently, the prognostic significance of these differentially expressed mRNAs was also 


\section{Cellular Physiology Cell Physiol Biochem 2018;50:473-488

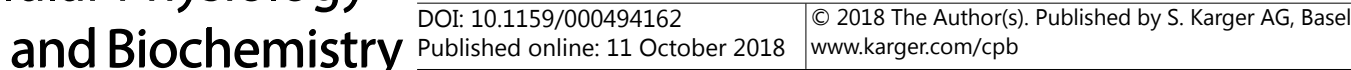

Yang et al.: Analysis of Expression Profile of Lncrnas with Cerna in TNBC

explored. The results revealed that 124 mRNAs were significantly associated with overall survival of patients with TNBC. Some of these mRNAs including WISP1, S1PR1 and SORBS1 have been characterized as oncogenes or tumor suppressors in various cancers. It has been showed that WISP1 gene might be a predictive marker of breast cancer metastasis [39]. Our data also indicated that WISP1 was inversely related to patients overall survival. Meanwhile, we found that SORBS1 could prolong the survival of TNBC patients. Lately, the research revealed that SORBS1 suppressed tumor metastasis and improved the sensitivity of cancer to chemotherapy drug in breast cancer. The researches suggest that these genes might play important regulatory roles in the tumorigenesis and development of TNBC and could serve as prognostic predictors for TNBC patients.

More and more dysregulated lncRNAs have been found in various human diseases, however, to date, only a few IncRNAs have been functionally characterized in cancers. Recently, lncRNAs have been found to function as ceRNAs to regulate gene expression. The researches demonstrated that dysregulated expressions of IncRNAs could significantly interfere with the balance of ceRNA network and influence miRNA-mediated cross-talk between lncRNA and mRNA, thus contributing to the initialization and development of cancers [40]. Here, we constructed a IncRNA-miRNA-mRNA ceRNA network based on the data derived from TCGA database in TNBC, which could provide important clues for the key RNAs in the development and progression of TNBC. The specific ceRNA network in TNBC includes 66 lncRNAs, 24 miRNAs and 55 mRNAs. For example, we predicted that LncRNA DLX6-AS1 could regulate the expressions of FSCN1, SOX11 and PSAT1 through competitive binding to hsa-miR-145. It has been reported that IncRNA DLX6-AS1 was up-regulated in lung adenocarcinoma (LAC) tissues compared to paired adjacent normal lung tissues and high DLX6-AS1 expression levels were significantly associated with both histological differentiation and TNM stage [41]. Recent researches have demonstrated that FSCN1, SOX11 and PSAT1 were associated with poor clinical outcome in breast cancer [42-44]. Our results are consistent with these reports.

In order to better understand the biological implication of the lncRNAs involved in the ceRNA network, KEGG pathway analysis was performed for the co-expressing protein coding genes in the network. The results showed that the target genes of miRNAs in ceRNA network are significantly enriched in cancer-related signaling pathways, such as microRNA in cancer, apoptosis, transcriptional misregulation in cancer, Ras signaling pathway and MAPK signaling pathway. The data suggested that these IncRNAs may participate in the tumorigenesis and development via regulating the cancer-related pathways in TNBC. Our data are in line with previous reports and provide a new therapeutic possibility for TNBC $[45,46]$, but further experimental validation is needed .

To further probe the correlations between the clinical characteristics and differentially expressed lncRNAs in the ceRNA network, tumor samples were divided into several subgroups according to TNM staging. The correlation analysis between clinical stage, pathology grade of TNBC and lncRNAs was executed. As a result, 9 lncRNAs including ST7-AS2, ST7-OT4, NLGN1-AS1, HOTTIP, LINC00491, C1orf143, C10orf126, C8orf31 and ELFN2 were found to be significantly associated with the TNM stage of TNBC. These data suggest their oncogenic roles in TNBC. Interestingly, our data further support the finding that HOTTIP expression was correlated with tumor size, lymph node status, TNM stage, overall survival and diseasefree survival in the breast cancer [26].

\section{Conclusion}

In conclusion, by means of comprehensive analysis of mRNA, IncRNA and miRNA expression profiles and clinical data of a large cohort of TNBC patients in TCGA database, we analyzed the expression profiles of lncRNAs and mRNAs in TNBC patients and identified TNBC-specific lncRNAs and miRNAs, which might be involved in the development, progression and prognosis of TNBC. More importantly, we successfully constructed a potential ceRNA 


\section{Cellular Physiology Cell Physiol Biochem 2018;50:473-488 \begin{tabular}{l|l|l} 
and Biochemistry Published online: 11 October 2018 & $\begin{array}{l}\text { @ } 2018 \text { The Author(s). Published by S. Karger AG, Basel } \\
\text { www.karger.com/cpb }\end{array}$ \\
\hline
\end{tabular}}

Yang et al.: Analysis of Expression Profile of Lncrnas with Cerna in TNBC

regulatory network in TNBC. The results provide novel insights into the mechanism underlying pathogenesis and development of TNBC. Our data might lay a foundation for further functional research of lncRNAs in TNBC and suggest that these specific lncRNAs and miRNAs could be valuable diagnostic, prognostic biomarkers and therapeutic targets for TNBC.

\section{Acknowledgements}

This work was supported by the National Natural Science Foundation of China (NO. 81672536) and the project of the top-notch talent cultivation program for the graduate students of Chongqing Medical University (NO. BJRC201711).

\section{Disclosure Statement}

None of the authors have conflicts of interest.

\section{References}

1 Dent R, Trudeau M, Pritchard KI, Hanna WM, Kahn HK, Sawka CA, Lickley LA, Rawlinson E, Sun P, Narod SA: Triple-Negative Breast Cancer: Clinical Features and Patterns of Recurrence. Clin Cancer Res 2018;13:4429-4434.

-2 Hua Z, Chen Z, Wang X, Huang Z, He Z, Chen Y: Long non-coding RNA: a new player in cancer. J Hematol Oncol 2013;6:37.

-3 Xiong XD, Ren X, Cai MY, Yang JW, Liu X, Yang JM: Long non-coding RNAs: An emerging powerhouse in the battle between life and death of tumor cells. Drug Resist Updat 2016;26:28-42.

4 Huarte M: The emerging role of lncRNAs in cancer. Nat Med 2015;21:1253.

-5 Zhang W, Shi S, Jiang J, Li X, Lu H, Ren F: LncRNA MEG3 inhibits cell epithelial-mesenchymal transition by sponging miR-421 targeting E-cadherin in breast cancer. Biomed Pharmacother 2017;91:312-319.

-6 Luo M, Li Z, Wang W, Zeng Y, Liu Z, Qiu J: Long non-coding RNA H19 increases bladder cancer metastasis by associating with EZH2 and inhibiting E-cadherin expression. Cancer Lett 2013;333:213-221.

7 Hirata H, Hinoda Y, Shahryari V, Deng G, Nakajima K, Tabatabai ZL, Ishii N, Dahiya R: Long noncoding RNA MALAT1 promotes aggressive renal cell carcinoma through Ezh2 and interacts with miR-205. Cancer Res 2015;75:1322.

8 Cai H, Yao J, An Y, Chen X, Chen W, Wu D, Luo B, Yang Y, Jiang Y, Sun D: LncRNA HOTAIR acts a competing endogenous RNA to control the expression of notch3 via sponging miR-613 in pancreatic cancer. Oncotarget 2017;8:32905-32917.

-9 Gang L, Miao W, Wu X, Dan T, Xiao X, Liang W, Fan M, Zeng F, Jiang G: Long Non-Coding RNA MEG3 Inhibits Cell Proliferation and Induces Apoptosis in Prostate Cancer. Cell Physiol Biochem 2015;37:2209.

10 Li Y, Li Y, Huang S, He K, Zhao M1, Lin H, Li D, Qian J, Zhou C, Chen Y, C H: Long non-coding RNA growth arrest specific transcript 5 acts as a tumour suppressor in colorectal cancer by inhibiting interleukin-10 and vascular endothelial growth factor expression. Oncotarget 2017;8:13690-13702.

$>11$ He A, Liu Y, Chen Z, Li J, Chen M, Liu L, Liao X, Lv Z, Zhan Y, Zhuang C: Over-expression of long noncoding RNA BANCR inhibits malignant phenotypes of human bladder cancer. J Exp Clin Cancer Res 2016;35:125.

-12 Cai Z, Cao R, Zhang K, Xue Y, Zhang C, Zhou Y, Zhou J, Sun H, Fu XD: Oncogenic miR-17/20a Forms a Positive Feed-forward Loop with the p53 Kinase DAPK3 to Promote Tumorigenesis. J Biol Chem 2015;290:1996719975.

13 Salmena L, Poliseno L, Tay Y, Kats L, Pandolfi PP: A ceRNA Hypothesis: The Rosetta Stone of a Hidden RNA Language? Cell 2011;146:353-358.

14 Chen Z, Liu H, Yang H, Gao Y, Zhang G, Hu J: The long noncoding RNA, TINCR, functions as a competing endogenous RNA to regulate PDK1 expression by sponging miR-375 in gastric cancer. Onco Targets Ther 2017;10:3353-3362. 


\section{Cellular Physiology Cell Physiol Biochem 2018;50:473-488 \begin{tabular}{l|l} 
DOI: 10.1159/000494162 & $\begin{array}{l}\text { O } 2018 \text { The Author(s). Published by S. Karger AG, Basel } \\
\text { www.karger.com/cpb }\end{array}$ \\
\hline and Biochemistry &
\end{tabular}

15 Huang G, Wu X, Li S, Xu X, Zhu H, Chen X: The long noncoding RNA CASC2 functions as a competing endogenous RNA by sponging miR-18a in colorectal cancer. Sci Rep 2016;6:26524.

-16 Oualla K, Elzawahry HM, Arun B, Reuben JM, Woodward WA, Eldin HG, Lim B, Mellas N, Ueno NT, Fouad TM: Novel therapeutic strategies in the treatment of triple-negative breast cancer. Ther Adv Med Oncol 2017;9:493-511.

17 Cabanski CR, White NM, Dang HX, Silvafisher JM, Rauck CE, Cicka D, Maher CA: Pan-cancer transcriptome analysis reveals long noncoding RNAs with conserved function. RNA Biol 2015;12:628-642.

18 Sun M, Nie F, Wang Y, Zhang Z, Hou J, He D, Xie M, Xu L, De W, Wang Z: LncRNA HOXA11-AS Promotes Proliferation and Invasion of Gastric Cancer by Scaffolding the Chromatin Modification Factors PRC2, LSD1, and DNMT1. Cancer Res 2016;76:6299-6310.

19 Terashima M, Tange S, Ishimura A, Suzuki T: MEG3 Long Noncoding RNA Contributes to the Epigenetic Regulation of Epithelial-Mesenchymal Transition in Lung Cancer Cell Lines. J Biol Chem 2016;292:82.

20 Xi J, Feng J, Li Q, Li X, Zeng S: The long non-coding RNA lncFOXO1 suppresses growth of human breast cancer cells through association with BAP1. Int J Oncol 2017;50:1663.

21 Huang J, Zhou N, Watabe K, Lu Z, Wu F, Xu M, Mo YY: Long non-coding RNA UCA1 promotes breast tumor growth by suppression of p27 (Kip1). Cell Death Dis 2014;5:e1008.

22 Xu S, Kong D, Chen Q, Ping Y, Pang D: Oncogenic long noncoding RNA landscape in breast cancer. Mol Cancer 2017;16:129.

23 Xue X, Yang YA, Zhang A, Fong KW, Kim J, Song B, Li S, Zhao JC, J Y: LncRNA HOTAIR enhances ER signaling and confers tamoxifen resistance in breast cancer. Oncogene 2016;35:2746-2755.

-24 Yun Z, Wang C, Xia L, Wu C, Yin H: Long non-coding RNA HOTAIR enhances radioresistance in MDA-MB231 breast cancer cells. Oncol Lett 2017;13:1143-1148.

25 Li W, Jia G, Qu Y, Du Q, Liu B, Liu B: Long Non-Coding RNA (LncRNA) HOXA11-AS Promotes Breast Cancer Invasion and Metastasis by Regulating Epithelial-Mesenchymal Transition.Med Sci Monit 2017;23:33933403.

26 Yang Y, Qian J, Xiang Y, Chen Y, Qu J: The prognostic value of long noncoding RNA HOTTIP on clinical outcomes in breast cancer. Oncotarget 2017;8:6833-6844.

27 Giza DE, Vasilescu C, Calin GA: MicroRNAs and ceRNAs: therapeutic implications of RNA networks. Expert Opin Biol Ther 2014;14:1285-1293.

28 Shan Y, Liu Y, Zhao L, Liu B, Li Y, Jia L: MicroRNA-33a and let-7e inhibit human colorectal cancer progression by targeting ST8SIA1. Int J Biochem Cell Biol 2017;90:48-58.

29 Bar I, Merhi A, Abdel-Sater F, Ben Addi A, Sollennita S, Canon JL, P D: The microRNA miR-210 is expressed by cancer cells but also by the tumor microenvironment in triple-negative breast cancer. J Histochem Cytochem 2017;65:335-346.

30 Lü L, Mao X, Shi P, He B, Xu K, Zhang S, Wang J: MicroRNAs in the prognosis of triple-negative breast cancer: A systematic review and meta-analysis. Medicine (Baltimore) 2017;96:e7085.

-31 Zhang Y, Tang X, Shi M, Wen C, Shen B: MiR-216a decreases MALAT1 expression, induces G2/M arrest and apoptosis in pancreatic cancer cells. Biochem Biophys Res Commun 2017;483:

-32 Zhang D, Zhao L, Shen Q, Lv Q, Jin M, Ma H, Nie X, Zheng X, Huang S, Zhou P: Down-regulation of KIAA1199/ CEMIP by miR-216a suppresses tumor invasion and metastasis in colorectal cancer. Int J Cancer 2017;140:2298-2309.

-33 Koduru SV, Tiwari AK, Leberfinger A, Hazard SW, Kawasawa YI, Mahajan M, Ravnic DJ: A Comprehensive NGS Data Analysis of Differentially Regulated miRNAs, piRNAs, IncRNAs and sn/snoRNAs in Triple Negative Breast Cancer. J Cancer 2017;8:578.

-34 Wang J, Tsouko E, Jonsson P, Bergh J, Hartman J, Aydogdu E, Williams C: miR-206 inhibits cell migration through direct targeting of the actin-binding protein Coronin $1 \mathrm{C}$ in triple-negative breast cancer. Mol Oncol 2014;8:1690.

35 Albiges L, Goubar A, Scott V, Vicier C, Lefèbvre C, Alsafadi S, Commo F, Saghatchian M, Lazar V, Dessen P: Chk1 as a new therapeutic target in triple-negative breast cancer. Breast 2014;23:250-258.

-36 Ting CH, Chen YC, Wu CJ, Chen JY: Targeting FOSB with a cationic antimicrobial peptide, TP4, for treatment of triple-negative breast cancer. Oncotarget 2016;7:40329-40347.

-37 Huang M, Zhong Z, Lv M, Shu J, Tian Q, Chen J: Comprehensive analysis of differentially expressed profiles of IncRNAs and circRNAs with associated co-expression and ceRNA networks in bladder carcinoma. Oncotarget 2016;7:47186-47200. 


\section{Cellular Physiology Cell Physiol Biochem 2018;50:473-488

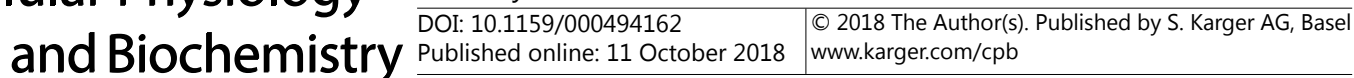 \\ Yang et al.: Analysis of Expression Profile of Lncrnas with Cerna in TNBC}

38 Chen C, Li Z, Yang Y, Xiang T, Song W, S L: Microarray expression profiling of dysregulated long non-coding RNAs in triple-negative breast cancer. Cancer Biol Ther 2015;16:856-865.

-39 Taghavi A, Akbari ME, Hashemi-Bahremani M, Nafissi N, Khalilnezhad A, Poorhosseini SM, Hashemi-Gorji F, VR Y: Gene expression profiling of the 8q22-24 position in human breast cancer: TSPYL5, MTDH, ATAD2 and CCNE2 genes are implicated in oncogenesis, while WISP1 and EXT1 genes may predict a risk of metastasis. Oncol Lett 2016;12:3845-3855.

40 Liu L, Yang J, Zhu X, Li D, Lv Z, Zhang X: Long noncoding RNA H19 competitively binds miR-17-5p to regulate YES1 expression in thyroid cancer. FEBS J 2016;283:2326-2339.

41 Li J, Li P, Zhao W, Yang R, Chen S, Bai Y, Dun S, Chen X, Du Y, Wang Y: Expression of long non-coding RNA DLX6-AS1 in lung adenocarcinoma. Cancer Cell Int 2015;15:48.

42 Wu Q, Yan H, Tao SQ, Wang XN, Mou L, Chen P, Cheng XW, Wu WY, Wu ZS: XIAP 3'-untranslated region as a ceRNA promotes FSCN1 function in inducing the progression of breast cancer by binding endogenous miR29a-5p. Oncotarget 2017;8:16784.

43 Oliemuller E, Kogata N, Bland P, Kriplani D, Daley F, Haider S, Shah V, Sawyer E, Howard B: SoX11 promotes invasive growth and ductal carcinomain situprogression. J Pathol 2017;243:193-207.

-44 Marchi TD, Timmermans MA, Sieuwerts AM, Smid M, Look MP, Grebenchtchikov N, Sweep FCGJ, Smits JG, Magdolen V, Deurzen CHMV: Phosphoserine aminotransferase 1 is associated to poor outcome on tamoxifen therapy in recurrent breast cancer. Sci Rep 2017;7:2099.

45 Wang X, Zhang W, Tang J, Huang R, Li J, Xu D, Xie Y, Jiang R, Deng L, Zhang X: LINC01225 promotes occurrence and metastasis of hepatocellular carcinoma in an epidermal growth factor receptor-dependent pathway. Cell Death Dis 2016;7:e2130.

46 Gan Y, Han N, He X, Yu J, Zhang M, Zhou Y, Liang H, Deng J, Zheng Y, Ge W, Long Z, X X: Long non-coding RNA CASC2 regulates cell biological behaviour through the MAPK signalling pathway in hepatocellular carcinoma. Tumour Biol 2017;39:1010428317706229. 